\title{
Functional Properties of Monolayer and Bilayer Graphene Hall-Effect Sensors
}

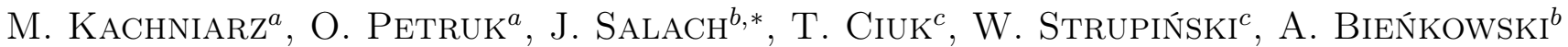 \\ AND R. SZEWCZYK ${ }^{b}$ \\ ${ }^{a}$ Industrial Research Institute for Automation and Measurements, Al. Jerozolimskie 202, 02-486 Warsaw, Poland \\ ${ }^{b}$ Institute of Metrology and Biomedical Engineering, Warsaw University of Technology, \\ sw. A. Boboli 8, 02-525 Warsaw, Poland \\ ${ }^{c}$ Institute of Electronic Materials Technology, Wólczyńska 133, 01-919 Warsaw, Poland
}

\begin{abstract}
The paper describes the design, development, and investigation of a new type of Hall-effect sensors of a magnetic field made of graphene. The epitaxial growth of high-quality graphene structures was performed using a standard hot-wall CVD reactor, which allows for easy integration with an existing semiconductors production technologies. The functional properties of developed Hall-effect sensors based on graphene were investigated on special experimental setup utilizing Helmholtz coils as a source of reference magnetic field. Monolayer and quasifree-standing bilayer graphene structures were tested. Results presented in the paper indicate that graphene is very promising material for development of Hall-effect sensors. Developed graphene Hall-effect sensor exhibit highly linear characteristics and high magnetic field sensitivity.
\end{abstract}

DOI: 10.12693/APhysPolA.131.1250

PACS/topics: 07.55.Ge, 85.75.Ss, 85.30.Fg, 81.05.ue, 73.22.Pr, 72.80.Vp

\section{Introduction}

The Hall effect is connected with the generation of a voltage potential (the Hall voltage) across a currentcarrying conductive plate, when it is subjected to the magnetic field perpendicular to the current flow direction [1]. The Hall voltage $V_{H}$ is determined by carrier concentration in the plate $n$ and is proportional both to the current $I$ and strength of the magnetic field $B$ [2]:

$$
V_{H}=-\frac{I B}{n t e},
$$

where $t$ is the thickness of the Hall-effect structure and $e$ is the electron elementary charge.

The Hall effect sensors dominate the market of industrial measurement of magnetic fields, which is worth about 1 billion dollars [3]. For this reason, such sensors are intensively developed. This development is focused on novel materials with higher electron mobility, enabling higher sensitivity of the sensors. One of the newest materials considered for the Hall-effect sensors is graphene, 2-dimensional allotrope of carbon. Due to a high electron mobility and carrier concentration graphene is expected to be a good material for the Hall-effect sensors.

The following paper presents the results of investigation on the graphene Hall-effect sensors made of the monolayer and bilayer graphene structures. Such sensors may be widely applied as sensing elements of the precise current transformers or sensing elements in the magnetovision systems for non-destructive testing [4].

*corresponding author; e-mail: j.salach@mchtr.pw.edu.pl

\section{Preparation}

of the graphene Hall-effect structure

Monolayer and quasi-free-standing bilayer graphene structures were grown using the chemical vapor deposition (CVD) method on the Si face of semi-insulating on-axis $4 \mathrm{H}-\mathrm{SiC}(0001)$ substrates in a standard hot-wall CVD Aixtron VP508 reactor. Prior to the growth, in situ etching of the $\mathrm{SiC}$ surface was carried out in hydrogen atmosphere. The epitaxial CVD growth of graphene was realized under dynamic flow conditions that simultaneously inhibit $\mathrm{Si}$ sublimation and promote the mass transport of propane molecules to the $\mathrm{SiC}$ substrate [5].

Monolayer graphene grown on the $\mathrm{Si}$ face of $\mathrm{SiC}$ rests on a buffer layer, which is the first layer of carbon atoms covalently bound to the substrate $[6-8]$. It can be decoupled to form a quasi-free-standing bilayer graphene (QFS-bilayer) through in situ hydrogen atoms intercalation [9]. In this paper, hydrogen intercalation was carried out at $1000^{\circ} \mathrm{C}$ in $90 \mathrm{kPa} \mathrm{Ar}$ atmosphere. The intercalating atoms diffuse underneath the buffer layer and bind themselves to the topmost $\mathrm{Si}$ atoms of the $\mathrm{SiC}$ substrate, converting the buffer layer to a mostly $s p^{2}$-hybridized monolayer graphene. The resultant QFSbilayer graphene is partly screened from the substrate and exhibits on average three times higher carrier mobility than the non-intercalated one. Importantly, its transport properties are not degraded up to $700^{\circ} \mathrm{C}$.

The as grown samples were characterized by roomtemperature Hall effect measurements in the Van der Pauw geometry (0.55 T Ecopia HMS-3000 setup) with the four golden probes placed in the corners of the $10 \mathrm{~mm} \times 10 \mathrm{~mm}$ substrates. Monolayer graphene exhibits electron transport, whereas the QFS-bilayer exhibits hole 
transport. Typically, electron concentration in monolayer graphene spans the range of $2-9 \times 10^{12} \mathrm{~cm}^{-2}$ with their mobility up to $2000 \mathrm{~cm}^{2} /(\mathrm{V} \mathrm{s})$. This type of graphene is characterized by a distinctive mobility concentration interchange ability and the highest mobility is observed at the lowest carrier concentrations. In the QFS-bilayer graphene structure, the hole concentration is usually limited to $1-2 \times 10^{13} \mathrm{~cm}^{-2}$. The highest as grown mobility $\left(5300 \mathrm{~cm}^{2} /(\mathrm{V} \mathrm{s})\right)$ is obtained, when growth conditions promote carrier concentration of approximately $1.2 \times 10^{13} \mathrm{~cm}^{-2}$.

The samples were subsequently photolitographically patterned to form symmetrical equal-arm graphene Hall crosses with cross bar length being three times its width. $20 \mathrm{~nm} \mathrm{Ti} / 80 \mathrm{~nm} \mathrm{Au}$ ohmic contacts were e-beam deposited (Fig. 1). The Hall voltage was measured on one pair of contacts, whereas the current flow was implemented between the other two opposite contacts.

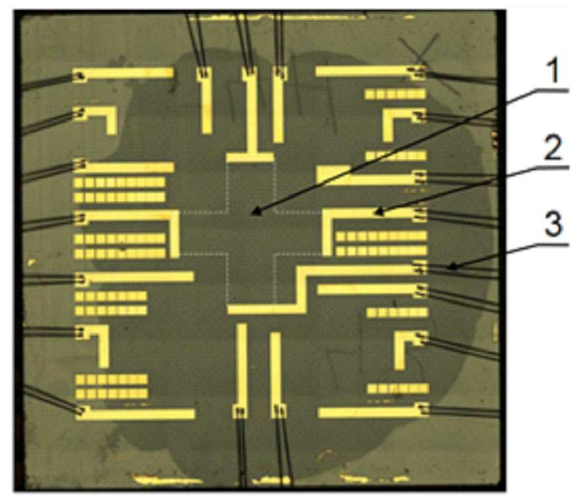

Fig. 1. Close-up view of the developed graphene based Hall-effect sensor: 1 - graphene cross (transparent), 2 — an electrode, 3 - bonding.

The dimensions of the cross arm were $700 \mu \mathrm{m} \times$ $2100 \mu \mathrm{m}$. Transport parameters of the structures are presented in Table I.

TABLE I

Transport properties of used graphene measured after processing.

\begin{tabular}{|c|c|c|c|}
\hline \multirow[b]{2}{*}{ Sample } & \multicolumn{2}{|c|}{ Carrier } & \multirow{2}{*}{$\begin{array}{l}\text { Type of } \\
\text { conduct }\end{array}$} \\
\hline & mob. $\left[\mathrm{cm}^{2} /(\mathrm{Vs})\right]$ & conc. $\left[\mathrm{cm}^{-2}\right]$ & \\
\hline & 3960 & $2.04 \times 10^{11}$ & $n$ \\
\hline QFS-bilayer & 2010 & $1.96 \times 10^{13}$ & $p$ \\
\hline
\end{tabular}

\section{Experimental setup}

Functional properties of developed Hall-effect sensors based on graphene were tested on a special computer controlled experimental setup, in which high precision Helmholtz coils were used as a source of reference magnetic field. The schematic diagram of the experimental setup is presented in Fig. 2.

Power for the Helmholtz coils was supplied by a Kepco regulated power supply to produce magnetic field acting

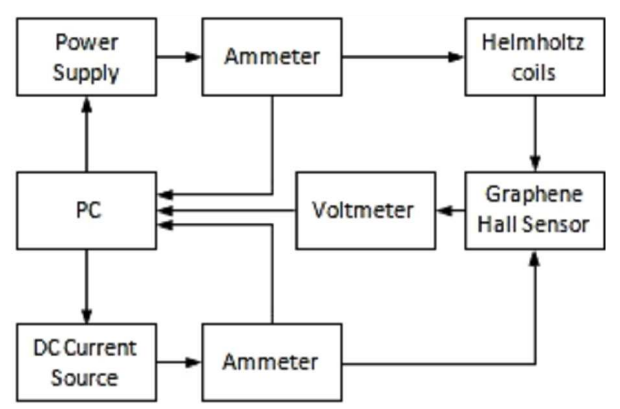

Fig. 2. Schematic block diagram of the experimental setup.

upon the investigated structure in the range of $\pm 6 \mathrm{mT}$. The current in the Helmholtz coils was measured by reference Appa 207 multimeter. The Helmholtz coils, as a magnetic field standard, need precise current measurement, as the field is directly proportional to it. Moreover, a Lakeshore 455 gaussmeter was used to measure this field in situ, for rough verification.

The sample was positioned in the middle of the Helmholtz coils, with a graphene Hall structure perpendicular to the magnetic field lines.

The Hall-effect structure was powered directly by the Motech laboratory power supply, which set the bias current in the sample. The bias current was measured simultaneously by the Metrol DM-22 multimeter. For highprecision Hall voltage measurement, the Fluke 8808A voltmeter was used. The Hall effect structures were tested under normal laboratory conditions, i.e. room temperature and air under normal pressure. There was no vacuum compartment or other environmental shielding of the samples.

\section{Results and discussion}

In order to compare the functional parameters of the Hall-effect sensors, monolayer and QFS-bilayer graphene structures were tested. During the investigation, samples were under the influence of constant magnetic field in the range of about $\pm 6 \mathrm{mT}$.

The characteristic of the Hall voltage $V_{H}$ as a function of the magnetic field $B$ obtained for the monolayer graphene structures is presented in Fig. 3.

The offset voltage was measured and compensated before the measurement of the Hall voltage (Table II). A similar characteristic for QFS-bilayer graphene sensor is presented in Fig. 4. Both sensors exhibit high linearity of the characteristic, which is confirmed by a high value of the linear determination coefficient $R^{2}$

The adequate parameter to characterize the currentdriven Hall-effect sensors is the current related sensitivity $S_{i}[10]$ :

$$
S_{i}=\frac{1}{I_{s}} \frac{\mathrm{d} V_{H}}{\mathrm{~d} B} .
$$

The current related sensitivity of the investigated graphene structures was measured experimentally. The 


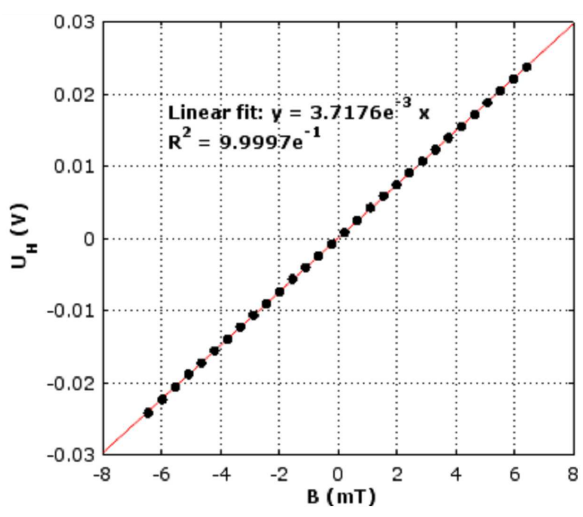

Fig. 3. Characteristic of developed monolayer graphene Hall-effect sensor at supply current $I_{s}=1200 \mu \mathrm{A}$.

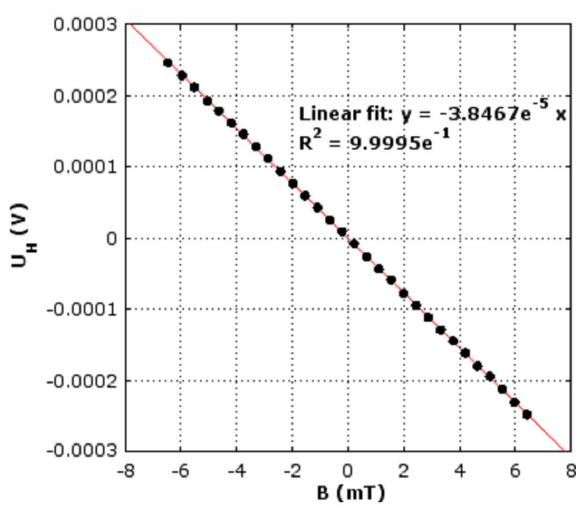

Fig. 4. Characteristic of developed QFS-bilayer graphene Hall-effect sensor at supply current $I_{s}=1200 \mu \mathrm{A}$.

current related sensitivity of the monolayer graphene structures was $S_{i}=3100 \mathrm{~V} /(\mathrm{A} \mathrm{T})$, whereas for the QFSbilayer graphene the value of current related sensitivity was $S_{i}=-32 \mathrm{~V} /(\mathrm{A} \mathrm{T})$. It is clearly seen that for the monolayer samples the sensitivity was two orders of magnitude higher than for the QFS-bilayer samples. Such a big difference results from the transport parameters of the materials tested. The parameters were worse for the QFS-bilayer graphene from the Hall-effect structure construction point of view, that is, the carrier density was higher, and the carrier mobility distinctly lower than for the monolayer graphene. The main difference between the materials is the type of electric charge transportation, hence the different sign of the characteristics slope (and of the sensitivity) [11].

TABLE II

Functional properties of the developed graphene Halleffect sensors at supply current $I_{s}=1200 \mu \mathrm{A}$

\begin{tabular}{|c|c|c|c|}
\hline Sample & $\begin{array}{l}\text { Current related } \\
\text { sens. }[\mathrm{V} / \mathrm{AT}]\end{array}$ & $\begin{array}{l}\text { Offset } \\
\text { volt. [V] }\end{array}$ & $\begin{array}{l}\text { Measured } \\
\text { res. }[\mu \mathrm{T}]\end{array}$ \\
\hline monolayer & 3100 & 1.170 & 46 \\
\hline QFS-bilayer & -32 & 0.012 & 118 \\
\hline
\end{tabular}

The ultra-sensitivity for the monolayer sensor was achieved, higher than previously presented $[12,13]$. It was possible through the use of $\mathrm{SiC}$ epitaxial technology that produces a high-quality graphene.

It is problematic that the offset voltage is quite high. The offset voltage has a decisive importance for the Halleffect sensors construction. The materials, from which the Hall-effect sensors are made have to be homogeneous in the whole volume - or whole area in the case of graphene.

This is due to inaccuracies in geometry reproduction of the Hall-effect structure, and due to substrate surface inhomogeneities resulting from the existence of terraces, which are specific for SiC. There is also the possibility of photoresist influence on the offset voltage.

Unfortunately, for the existing graphene production technologies, the existence of the additional layer seeds on the main layer is characteristic. Relative seeds area can be as high as 5\% [14] of the total graphene area. Their presence causes conductance heterogeneity and the effect of asymmetric voltage distribution in the Hall structure during the current flow.

Another very important parameter, from the point of view of metrology, is the resolution. Assuming that the main source of noise is thermal noise, defined as:

$$
V=\sqrt{4 k_{\mathrm{B}} T \Delta f R_{\text {out }}},
$$

where $k_{\mathrm{B}}$ is the Boltzmann constant, $T$ is temperature, $\Delta f$ is the bandwidth over which the noise is measured and $R_{\text {out }}$ is output resistance of the Hall-effect structure.

The Hall voltage for given driving current

$$
V=S_{i} I_{s} B \text {. }
$$

The dependence on the minimum value of the field distinguishable by the sensor can be derived

$$
B_{\min }=\frac{\sqrt{4 k_{\mathrm{B}} T \Delta f R_{\text {out }}}}{S_{i} I_{s}} .
$$

Substituting the numerical values from Table II into Eq. (5) and assuming temperature $T=300 \mathrm{~K}$, frequency bandwidth $\Delta f=1 \mathrm{kHz}$ and resistance $R_{\text {out }}$ for monolayer $12180 \Omega$ and $370 \Omega$ for QFS-bilayer graphene, we obtain the $B_{\min }=4 \mathrm{nT}$ and $B_{\min }=65 \mathrm{nT}$, accordingly.

In order to analyze the impact of transport parameters on the sensitivity of the device, a simple transformation of the above equations is made. Knowing that the resistance $R_{\text {out }}$ is inversely proportional to the conductivity, and that the sensitivity $S_{i}$ is inversely proportional to the charge carrier concentration $n$, additionally omitting the effect of geometry we obtain

$$
B_{\min } \approx \frac{\sqrt{T n}}{I_{s} \sqrt{\mu}} .
$$

Transport properties of graphene have a direct impact on the resolution of the Hall-effect sensors. The abovementioned dependences are directly pointing to the advantages of graphene in the construction of Hall sensors, and that for the monolayer graphene the $B_{\min }$ value is lower. 
The time changes of $U-I$ characteristics were measured for the selected graphene Hall-effect structure at several different values of supply current $I_{s}$. The results are presented in Figs. 5-7. The diagrams show a fair degree of noise of thermal origin for small currents. With an increasing value of the supply current, the average value of the noise waveform is also increasing while the noise amplitude remains almost unaffected.

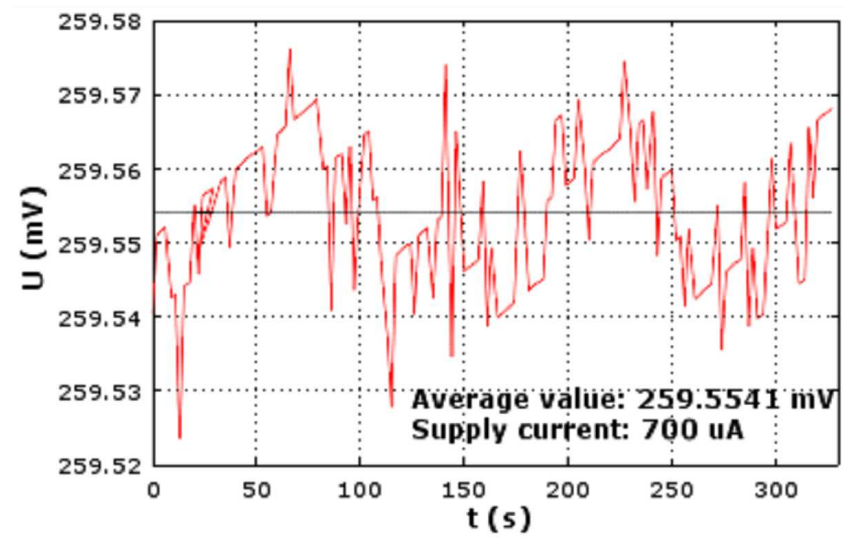

Fig. 5. $U-I$ characteristic of the developed graphene Hall-effect sensor in time for supply current $I_{s}=700 \mu \mathrm{A}$.

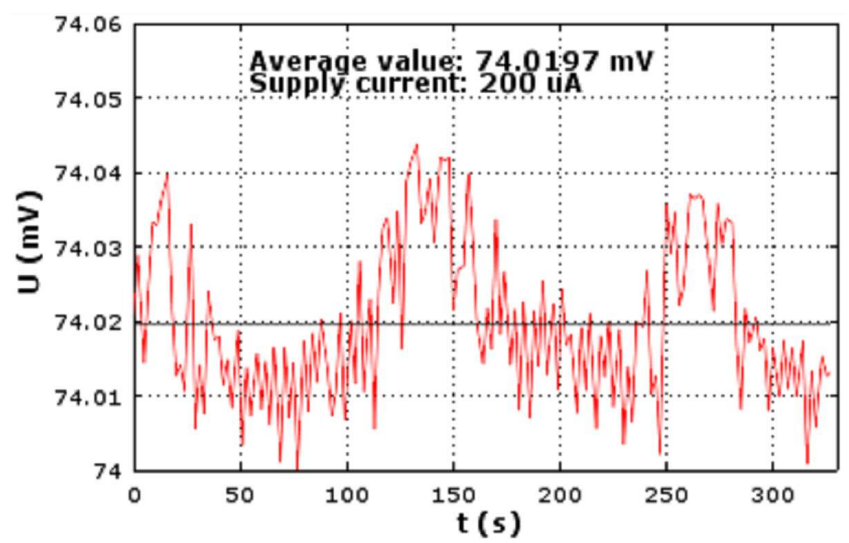

Fig. 6. As in Fig. 5 for supply current $I_{s}=200 \mu \mathrm{A}$.

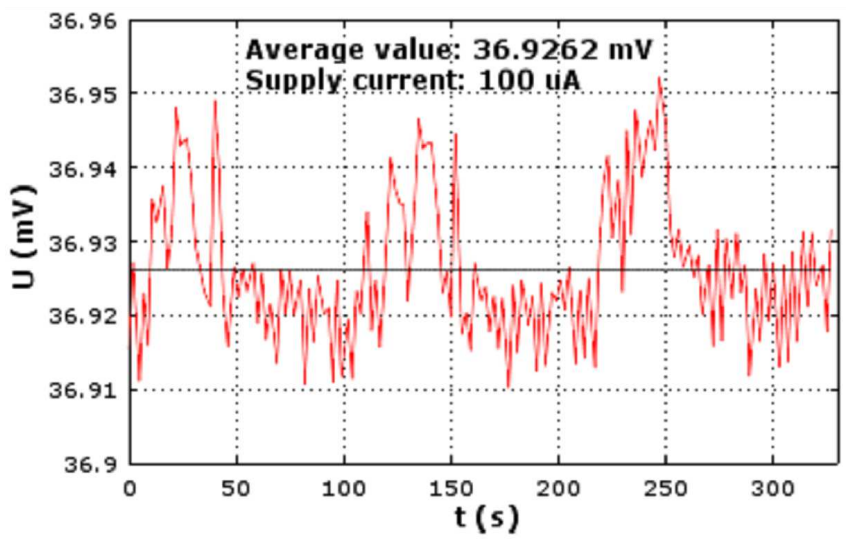

Fig. 7. As in Fig. 5 for supply current $I_{s}=100 \mu \mathrm{A}$.

\section{Conclusions}

The results presented in the article show that graphene is a very good material for applications in the Hall-effect sensors. Sensors made of monolayer graphene are characterized by much higher sensitivity than sensors made of other materials. The worse parameters of the QFSbilayer graphene sensors, in comparison with the monolayer sensors, are the effect of the lower charge carrier concentration. Also resolution of the monolayer graphene sensor is better than in the QFS-bilayer structure. On the other hand, the monolayer structure exhibits much higher offset voltage, which is important problem in application of the developed sensors.

The thermal noise observable for the investigated sensors is also undesirable effect. However, the noise amplitude is relatively low and is not dependent on the supply current, which is very important from the applicability point of view.

\section{Acknowledgments}

This work was partially supported by the National Center for Research and Development (Poland) within the GRAF-TECH program and partially supported by statutory founds of Institute of Metrology and Biomedical Engineering, Warsaw University of Technology (Poland).

\section{References}

[1] P. Ripka, Magnetic Sensors and Magnetometers, Artech House, Boston 2001.

[2] E. Ramsden, Hall-Effect Sensors: Theory and Application, Newnes, Boston 2006.

[3] Global Magnetic Field Sensors Market by Product, Technology and Applications, 2011.

[4] M. Nowicki, R. Szewczyk, Materials 6, 5593 (2013).

[5] W. Strupinski, K. Grodecki, A. Wysmolek, R. Stepniewski, T. Szkopek, P.E. Gaskell, A. Grüneis, D. Haberer, R. Bozek, J. Krupka, J.M. Baranowski, Nano Lett. 11, 1786 (2011).

[6] N. Ray, S. Shallcross, S. Hensel, O. Pankratov, Phys. Rev. B 86, 125426 (2012).

[7] C. Riedl, U. Starke, J. Bernhardt, M. Franke, K. Heinz, Phys. Rev. B 76, 245406 (2007).

[8] K.V. Emtsev, F. Speck, Th. Seyller, L. Ley, J.D. Riley, Phys. Rev. B 77, 155303 (2008).

[9] C. Riedl, C. Coletti, T. Iwasaki, A.A. Zakharov, U. Starke, Phys. Rev. Lett. 103, 246804 (2009).

[10] R.S. Popovic, Hall Effect Devices, Institute of Physics Publ., London 2004.

[11] N.W. Ashcroft, N.D. Mermin, Solid State Physics, Holt, Rinehart and Winston, New York 1976.

[12] V. Panchal, K. Cedergren, R. Yakimova, A. Tzalenchuk, S. Kubatkin, O. Kazakova, J. Appl. Phys. 111, 07E509 (2012).

[13] H. Xu, Z. Zhang, R. Shi, H. Liu, Z. Wang, S. Wang, L.M. Peng, Sci. Rep. 3, 1207 (2013).

[14] Z. Ni, Y. Wang, T. Yu, Z. Shen, Nano Res. 1, 273 (2008). 